\title{
Pulmonary Rehabilitation with a Stabilometric Platform After Thoracic Surgery: A Preliminary Report
}

\author{
by \\ Dariusz Jastrzębski ${ }^{1}$, Aleksandra Żebrowska ${ }^{2}$, Sebastian Rutkowski ${ }^{3}$, \\ Anna Rutkowska ${ }^{3}$ Joanna Warzecha ${ }^{1}$, Bettina Ziaja ${ }^{1}$, Aleksandra Pałka , \\ Barbara Czyżewska4, Damian Czyżewski ${ }^{4}$, Dariusz Ziora ${ }^{1}$
}

The aim of this study was to evaluate the influence of exercises on a stabilometric platform on the physical fitness and mobility of patients with lung cancer after thoracic surgery. The Experimental Group included 22, and the Control Group consisted of 21 patients. All included patients were enrolled after thoracic surgery due to lung cancer. The Experimental and Control Groups were enrolled in a 3-week in-hospital pulmonary rehabilitation program. The Experimental Group additionally performed daily 20-min training sessions on a stabilometric platform. Agility and flexibility were assessed with the Fullerton test before and after rehabilitation in both groups, and quality of life was assessed with the SF-36 questionnaire. Exercise performance stated as a distance in a 6 min walking test (6MWT) significantly improved in both groups with a medium effect size. The results of the Fullerton test indicated improvements in flexibility in both groups after the completion of the program without a significant difference between the groups and with a small effect size. In the Experimental Group, the best results were observed in the Arm curl ( $p=$ $0.0001)$, Chair stand $(p=0.04)$, Up and go $(p=0.001)$ and Chair sit and reach $(p=0.0001)$ tasks. No deterioration in the quality of life was observed in the Experimental or the Control Group after the completion of the program. Betweengroup analyses revealed significant differences in the Role-Physical $(R P)(p=0.020)$ and Mental-Health $(M H)(p=$ 0.025) domains of the SF-36. The rehabilitation program with a stabilometric platform improved agility and flexibility of patients after thoracic surgery without an effect size or significant differences between the Experimental and Control Groups.

Key words: pulmonary rehabilitation, lung cancer, physical fitness, stabilometric platform.

\section{Introduction}

According to the incidence of disease, lung cancer is the second-most frequent cancer after breast cancer in women and the second-most frequent cancer after prostate cancer in men (Ferlay et al., 2015). Early diagnosis and new therapies together with the strong dynamics of newly occurring cases pertain to the growth of patients suffering from lung cancer. In Poland, there are over 22,000 registered patients with lung cancer (lung cancer in Poland - medical perspective). The majority of cases occur after the age of 50 , and approximately $50 \%$ of cases among both females and males are diagnosed after the age of 65 (Didkowska et al., 2015). The most effective method of cancer treatment is surgical intervention with the complete removal of the tumor and the surrounding pulmonary tissue

1 - Department of Lung Diseases and Tuberculosis, School of Medicine with the Division of Dentistry, Medical University of Silesia, Zabrze, Poland.

2- Department of Physiology, Academy of Physical Education in Katowice, Poland.

3 - Institute of Physiotherapy, Department of Physical Education and Physiotherapy, Opole University of Technology, Opole, Poland.

4- Department of Thoracosurgery, School of Medicine with the Division of Dentistry, Medical University of Silesia, Zabrze, Poland. 
(Vansteenkiste et al., 2013). As Mraz et al. (2005) indicated, it is possible to observe a modification in the body's center of gravity and a reduction of postural stability after thoracic surgery. These effects may be caused by impairment of the stability of the musculature due to the surgical procedure or due to postoperative pain syndromes (Mraz et al., 2005). The long-term pain experienced by patients after surgery causes body abnormalities and pathological postures. Patients subconsciously protect themselves against this pain. This situation results in overloading of other elements of the musculoskeletal system with time and disorders of body posture. Additionally, restrictions of the range of motion of the upper limb on the side of the surgery arise and provoke further body posture faults. Therefore, rehabilitation procedures after the thoracic surgery should include exercises that rectify movement coordination deficits and correct poor body posture. The stabilometric platform could be used for this purpose as it enables not only the assessment of both the static and dynamic variables responsible for maintaining body posture and balance, but also postural muscle training. The stabilometric platform was originally created for the rehabilitation of neurological patients (e.g., post-stroke, Parkinson's disease and multiple sclerosis patients) (Cattaneo et al., 2016). Currently, the stabilometric platform is commonly applied in highly specialized orthopedic rehabilitation wards as well as geriatric and posttraumatic or postoperative convalescence wards (Siekański, 2008). According to the authors' knowledge, there are currently no reports of assessments of the possibilities concerning the stabilometric platform and its use for the rehabilitation of postoperative thoracic patients.

The purpose of the study was to compare a standard rehabilitation program with the one including sessions with stabilometric platform exercises that also involved the use of a biofeedback method, and their influence on physical fitness levels of patients with pulmonary cancer who had undergone oncological thoracic surgery due to lung cancer.

\section{Methods}

\section{Participants}

All patients provided written consent to participate in the research. Patients were included in the study if they had the ability to perform the 6-minute walk test (6MWT), had an Eastern Cooperative Oncology Group (Oken et al., 1982) performance status of $0-1$, were able to complete questionnaires and were willing to participate in training. Patients were deemed not eligible if they had uncontrolled hypertension, unstable coronary artery disease, anemia (hemoglobin $<10 \mathrm{~g} / \mathrm{dl}$ ), severe osteoarthritis, bone or CNS metastases. The study was approved by the Ethics Committee of the Silesian Medical University (KNW/0022/KB1/184a/I/11/12).

The study included 43 inpatients who were admitted for a 2-week rehabilitation program in the Rehabilitation Ward of the Hospital. All patients were enrolled $10 \pm 4$ weeks after lobectomy due to lung cancer. The Experimental Group (EG) consisted of 22 patients (11 men and 11 women; age: $69.8 \pm 6.0$ years). The patients in the EG participated in standard pulmonary rehabilitation in addition to daily training using the stabilometric platform. The Control Group (CG) consisted of 21 patients (11 men and 10 women; age: $69.0 \pm 9.6$ years) who participated in a standard pulmonary rehabilitation program.

\section{Measures}

After receiving consent, the patients' medical records were reviewed to determine their eligibility. For all patients in the EG and CG, the following factors were assessed at baseline (Day 1) and after the completion of the experiment (Day 21):

- Exercise performance was assessed by the distance covered in the 6MWT, which was conducted according to Polish guidelines (Przybyłowski et al., 2015).

- To estimate the quality of life, the ShortForm Health Survey (SF-36) was applied. This survey belongs to a group of tools used to estimate the general quality of life and is commonly used in many health service institutions (Ware at al., 2004). The SF-36 consists of 36 questions that describe the state of health. The SF-36 allows for the description of the physical quality of life as the Physical Cumulative Score (PCS ), which consists of 4 domains: Physical Functioning (PF), i.e., limitations in selfcare activities from a low score: with 
limited performance of most of physical activities including bathing or dressing, to the high score: performance of most types of physical activities without limitations due to health; Role Physical (RP), i.e., problems with work or other daily activities as a result of physical health; Bodily Pain (BP), i.e. from very severe and extremely limiting pain to lack of pain or limitations due to pain; and General Health (GH), i.e. from poor to excellent. Additionally, the SF36 assesses the mental quality of life in term of two domains: Role Emotional (RE), i.e., problems with work or other daily activities as a result of emotional problems, and Mental Health ( $\mathrm{MH})$, i.e., from the feeling of anxiety and depression to the feeling of happiness and calmness (Ware et al., 2004). The cumulative point totals range from 0 to 100. More points are indicative of better outcomes. The examined patients individually completed the questionnaires. A non-commercial license agreement from the Office of Grants and Scholarly Research was applied (Optuminsight Life Sciences, Inc., license number: QM043126). The methodology and data analysis for the SF36 questionnaire had been previously described (Jastrzębski et al., 2005).

- Functional fitness was assessed with the Fullerton test (Rikli and Jones, 1999), which is composed of the following 6 components that evaluate upper and lower body strength, exercise performance, motor coordination, and balance:

1) The Chair Stand Test assesses lower body strength and considers the number of full stands completed in $30 \mathrm{~s}$ with the arms folded across the chest.

2) The Arm Curl test assesses upper body strength and considers the number of bicep curls that can be completed in $30 \mathrm{~s}$ by the dominant hand with a hand weight of 2.27 $\mathrm{kg}$ for woman and $3.36 \mathrm{~kg}$ for men.

3) The Chair Sit and Reach Test evaluates lower body flexibility. From a sitting position on a chair with legs extended forward, participants are required to reach forward toward the toes. The result was the distance $(\mathrm{cm})$ reached $(+$ or -$)$ between the extended fingers and tips of the toes.
4) The Up and Go test assesses coordination/agility/dynamic balance as the time (in seconds) needed to rise from a seated position, walk a distance of $2.44 \mathrm{~m}$, turn, and return to a seated position.

5) The Back Scratch test assesses upper shoulder flexibility. With one hand reaching over the shoulder and one up the middle of the back, the result is the number of centimeters between the extended middle fingers (+ or - ).

6) The final component is the 6MWT.

\section{Design and Procedures}

All patients (EG and CG) performed supervised exercise training at different intensities of particular components assigned based on the initial 6MWT result. The patients participated in the exercise sessions 5 times per week. The sessions consisted of the following: 1) $30 \mathrm{~min}$ of respiratory exercises that included relaxation exercises for the breathing muscles, strengthening exercises with resistance for the diaphragm, exercises to increase costal or chest breathing, a prolonged exhalation exercise, and chest percussion; 2) training on a cycle ergometer or treadmill for $20-30 \mathrm{~min}$ at an intensity of $30-80 \%$ of the peak work rate according to individual tolerance; 3) resistance exercise at an intensity of $40-70 \%$ of the 1-repetition maximum (1RM); or 4$)$ Nordic walking for 45 minutes (depending on the weather and health condition of the patient); and 6) relaxation training. The heart rate and $\mathrm{O}_{2}$ saturation were continuously monitored throughout training sessions by pulse oximetry (Konica Minolta Pulsox-300i Pulse Oximeter), and the blood pressure during exercise was intermittently assessed.

The Alfa stabilometric platform (AC International East) was used to conduct additional training in the EG to stimulate the musculoskeletal and nervous system responsible for controlling balance.

The exercises performed on the platform included the following:

- Pendulum (moving the center of pressure according to displayed patterns by walking between extreme positions)

- Paths (leaning the patient toward the displayed goals)

- Hunting (following an escaping point)

- Tracking (moving the center of pressure 
over a given circle)

- Colors (acquiring all white targets while avoiding the red ones).

The time allotted for each exercise on the stabilometric platform was 3 minutes, with the 1min rest interval between following exercises; a complete exercise session (1 per day) lasted 20 min. .

\section{Statistical Analysis}

The normality of the data was evaluated using the Shapiro-Wilk test. The Student's t-test was applied for normally distributed data with equal variances, while the Wilcoxon signed-rank test for the non-normally distributed data. Statistically significant values were defined at $p<$ 0.05 . Inter-group comparisons of the changes were performed with the Mann-Whitney U-test. The effect sizes (Cohen's d) of the differences were also calculated (small: $0 \leq|\mathrm{d}| \leq 0.5$; medium: $0.5<$ $|\mathrm{d}| \leq 0.8$; and large: $|\mathrm{d}|>0.8$ ).

\section{Results}

All 43 of the initially included patients completed the 2-week rehabilitation program and underwent the final assessment. No adverse effects induced by exercise effort or training on the stabilometric platform were observed.

Table 2 presents the Fullerton test results for the EG and CG before and after rehabilitation and comparisons of the power sizes between groups. The distance covered in the 6MWT significantly improved in the Experimental Group $(p=0.03$, effect size 0.67$)$ as well as the Control
Group ( $p=0.003$, effect size 0.77). The difference in the 6MWT between groups was not statistically significant $(p=0.576)$ with a small effect size (0.331). The results of the Fullerton test revealed improvements in the Arm Curl (EG: $p=0.0001$, CG: $p=0.0005$ ), Chair Stand (EG: $p=0.04$, CG: $p=$ 0.002), and Up and Go (EG: $p=0.001$, CG: $p=$ $0.006)$ tests in both the SG and CG. Yet, only the EG exhibited an improvement in the Chair Sit and Reach test (EG: $p=0.0001$, CG: $p=0.12$ ). The results of the Back Scratch test were similar at the beginning and the end of the study in both the EG and CG. No differences between the SG and CG were observed in any of the components of the Fullerton test.

With regard to the SF-36 questionnaire, according to the methodological guidelines, the observed differences did not meet the criteria for clinical significance in the studied population (Ware at al., 1993). However, statistically significant differences in the quality of life before and after rehabilitation were found in both groups (Table 4). The EG $(p=0.039)$ and CG $(p=0.001)$ exhibited statistically significant improvements in bodily pain (BP). Furthermore, the EG exhibited improvements in PF $(p=0.023)$ and PCS $(p=$ $0.047)$, while the CG in GH $(p=0.004)$, VT $(p=$ $0.015), \mathrm{MH}(p=0.001)$ and MCS $(p=0.010)$. Intergroup analyses revealed significant differences in the RP $(p=0.020)$ and $\mathrm{MH}(p=$ 0.025) domains.

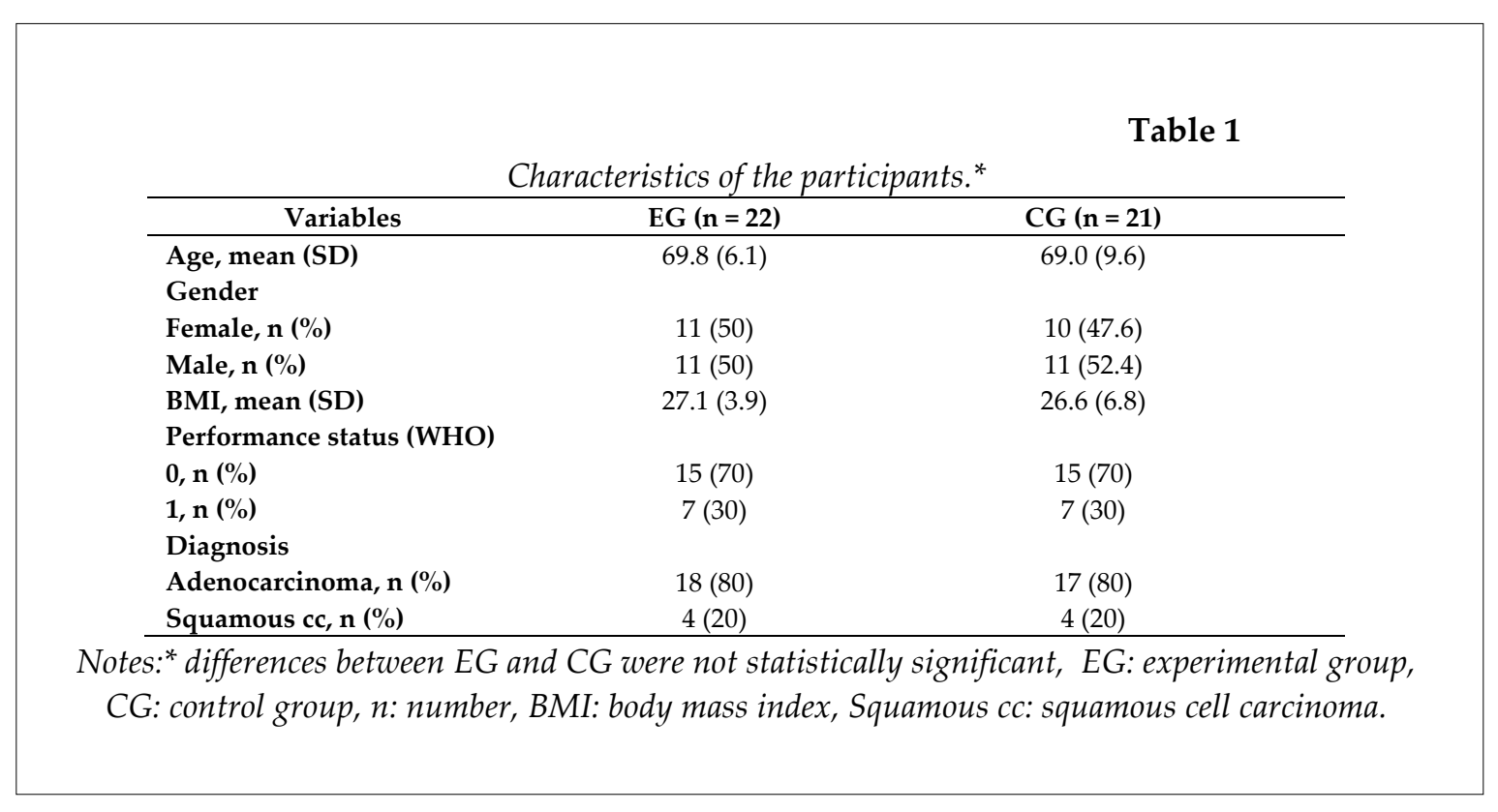


Table 2

Comparisons of the mean values from the Fullerton test

\begin{tabular}{|c|c|c|c|c|c|c|c|c|}
\hline & \multicolumn{3}{|c|}{ Experimental Group } & \multicolumn{5}{|c|}{ Control Group } \\
\hline & before PR & after PR & $p$ & $\begin{array}{c}\text { Cohen' } \\
\text { s d }\end{array}$ & before PR & after PR & $p$ & $\begin{array}{c}\text { Cohen' } \\
\text { s d }\end{array}$ \\
\hline $\begin{array}{l}\text { Arm curl } \\
\text { (rep.) }\end{array}$ & $11.8 \pm 3.3$ & $15.8 \pm 3.8$ & 0.001 & 1.33 & $11.6 \pm 3.5$ & $15.6 \pm 3.4$ & 0.001 & 1.33 \\
\hline $\begin{array}{l}\text { Chair stand } \\
\text { (rep.) }\end{array}$ & $9.8 \pm 2.5$ & $11.8 \pm 3.7$ & 0.040 & 0.78 & $9.0 \pm 2.9$ & $10.8 \pm 2.3$ & 0.002 & 0.50 \\
\hline $\begin{array}{l}\text { Chair sit and } \\
\text { reach }(\mathrm{cm})\end{array}$ & $-9.3 \pm 12.7$ & $\begin{array}{c}-6.2 \pm \\
11.2\end{array}$ & 0.001 & 0.26 & $-5.1 \pm 8.8$ & $-3.5 \pm 9.1$ & 0.120 & 0.23 \\
\hline $\begin{array}{l}\text { Up and go } \\
\text { (s) }\end{array}$ & $6.3 \pm 2.3$ & $8.1 \pm 3.0$ & 0.001 & 0.78 & $8.5 \pm 3.0$ & $6.9 \pm 2.4$ & 0.006 & 0.78 \\
\hline $\begin{array}{l}\text { Back scratch } \\
(\mathrm{cm})\end{array}$ & $-8.7 \pm 7.8$ & $-4.8 \pm 7.1$ & 0.090 & 0.57 & $-8.0 \pm 15.6$ & $-6.6 \pm 13.2$ & 0.230 & 0.14 \\
\hline 6MWT (m) & $\begin{array}{c}335.9 \pm \\
106.2\end{array}$ & $\begin{array}{c}401.8 \pm \\
90.9\end{array}$ & 0.030 & 0.67 & $\begin{array}{c}382.0 \pm \\
75.5\end{array}$ & $\begin{array}{l}439.6 \\
\pm 73.8\end{array}$ & 0.001 & 0.77 \\
\hline
\end{tabular}

Notes: PR: pulmonary rehabilitation, 6MWT: 6-minute walk test.

Table 3

Comparisons of the mean values from the Fullerton test between groups

\begin{tabular}{lcccc}
\hline & EG & CG & $p^{*}$ & $\begin{array}{c}\text { Observed } \\
\text { power }\end{array}$ \\
\hline Arm Curl (rep.) & $2.0 \pm 2.2$ & $1.7 \pm 2.2$ & 0.706 & 0.054 \\
Chair Stand (rep.) & $4.0 \pm 2.4$ & $4.0 \pm 2.9$ & 0.903 & 0.190 \\
$\begin{array}{l}\text { Chair Sit and Reach } \\
(\mathrm{cm})\end{array}$ & $3.1 \pm 4.1$ & $1.5 \pm 3.5$ & 0.343 & 0.143 \\
Up and go (s) & $1.8 \pm 1.8$ & $1.4 \pm 1.6$ & 0.743 & 0.313 \\
Back Scratch (cm) & $3.9 \pm 6.0$ & $1.4 \pm 5.1$ & 0.388 & 0.087 \\
6MWT (m) & $65.9 \pm 58.9$ & $57.6 \pm 60.8$ & 0.576 & 0.331 \\
\hline
\end{tabular}

Notes: * Mann-Whitney U-test, EG: experimental group,

CG: control group, 6MWT: 6-minute walk test. 
Table 4

Comparisons of the mean values from the SF-36 test

\begin{tabular}{|c|c|c|c|c|c|c|c|c|}
\hline & \multicolumn{4}{|c|}{ Experimental Group } & \multicolumn{4}{|c|}{ Control Group } \\
\hline & before PR & after PR & $p$ & $\begin{array}{c}\text { Cohen's } \\
\mathrm{d}\end{array}$ & before PR & after PR & $p$ & $\begin{array}{c}\text { Cohen's } \\
\text { d }\end{array}$ \\
\hline PF & $36.4 \pm 10.1$ & $39.3 \pm 8.7$ & 0.023 & 0.30 & $40.4 \pm 9.0$ & $44.9 \pm 14.6$ & 0.000 & 0.37 \\
\hline RP & $34.1 \pm 9.8$ & $36.8 \pm 8.0$ & 0.061 & 0.29 & $40.1 \pm 11.7$ & $36.6 \pm 8.2$ & 0.132 & -0.34 \\
\hline BP & $40.3 \pm 8.5$ & $43.1 \pm 9.7$ & 0.039 & 0.30 & $36.2 \pm 7.1$ & $41.7 \pm 7.8$ & 0.002 & 0.73 \\
\hline GH & $41.4 \pm 9.0$ & $41.0 \pm 6.9$ & 0.831 & -0.05 & $38.2 \pm 9.6$ & $41.0 \pm 9.2$ & 0.004 & 0.29 \\
\hline VT & $48.0 \pm 10.2$ & $48.1 \pm 8.3$ & 0.941 & 0.01 & $44.1 \pm 10.7$ & $48.1 \pm 8.9$ & 0.015 & 0.41 \\
\hline SF & $41.1 \pm 7.7$ & $43.6 \pm 9.8$ & 0.223 & 0.28 & $37.2 \pm 10.1$ & $38.3 \pm 11.6$ & 0.616 & 0.10 \\
\hline RE & $41.1 \pm 12.4$ & $41.7 \pm 11.0$ & 0.999 & 0.05 & $39.6 \pm 13.0$ & $40.0 \pm 10.6$ & 0.762 & 0.03 \\
\hline MH & $44.5 \pm 10.7$ & $48.6 \pm 8.3$ & 0.082 & 0.42 & $40.5 \pm 10.2$ & $47.3 \pm 9.1$ & 0.001 & 0.69 \\
\hline PCS & $36.4 \pm 9.1$ & $38.3 \pm 7.85$ & 0.047 & 0.22 & $38.5 \pm 4.9$ & $39.7 \pm 6.8$ & 0.213 & 0.20 \\
\hline MSC & $46.7 \pm 10.2$ & $48.4 \pm 7.74$ & 0.381 & 0.19 & $41.3 \pm 10.43$ & $45.3 \pm 9.4$ & 0.010 & 0.40 \\
\hline
\end{tabular}

Notes: PR: pulmonary rehabilitation, PF: physical functioning, RP: role physical, BP: bodily pain, GH: general health, VT: vitality, SF: social functioning, RE: role emotional, $M H$ : mental health, PCS: physical health, MSC: mental health.

Table 5

Comparisons of the mean values from the SF-36 test between groups

\begin{tabular}{ccccc}
\hline PF & EG & CG & $p^{*}$ & $\begin{array}{c}\text { Observed } \\
\text { power }\end{array}$ \\
\hline RP & $7.5 \pm 14.5$ & $6.1 \pm 15.1$ & 0.504 & 0.073 \\
BP & $7.4 \pm 17.5$ & $-4.2 \pm 19.9$ & 0.020 & 0.960 \\
GH & $1.0 \pm 14.9$ & $2.0 \pm 12.5$ & 0.874 & 0.061 \\
VT & $0.2 \pm 11.1$ & $4.3 \pm 6.1$ & 0.117 & 0.256 \\
SF & $2.3 \pm 16.2$ & $7.9 \pm 12.2$ & 0.215 & 0.437 \\
RE & $5.1 \pm 23.4$ & $0.5 \pm 18.7$ & 0.653 & 0.314 \\
MH & $1.5 \pm 25.3$ & $3.1 \pm 22.9$ & 1.000 & 0.080 \\
PCS & $3.4 \pm 18.0$ & $10.3 \pm 13.7$ & 0.025 & 0.597 \\
MSC & $3.7 \pm 9.7$ & $5.0 \pm 12.6$ & 0.780 & 0.070 \\
\hline
\end{tabular}

Notes: * Mann-Whitney U-test, EG: experimental group, CG: control group 


\section{Discussion}

The growing population of cancer survivors entails an urgent need to define clinical interventions that would optimize their functioning. Symptom management over the course of cancer treatment and throughout the lifespan of survivorship is noted to be one of the most significant challenges faced by both patients and healthcare providers. Exercise interventions have a strong evidence base that supports their inclusion in most cancer care plans. Surgically treated NSCLC (non-small cell lung cancer) patients are likely to have not only reduced cardiorespiratory capacity due to the removal of at least one lobe, but they also experience pain, coughing and reduced levels of daily activities following surgery. There is a strong rationale for considering people with NSCLC for referral to postoperative pulmonary rehabilitation programs (Holland et al., 2013; Stout et al., 2017).

Biofeedback has been used for more than fifty years in rehabilitation to facilitate acquisition of normal movement patterns after injury. Biofeedback is a technique in which biological information that would otherwise be unknown is provided to patients in real time. Providing patients and clinicians with biofeedback during rehabilitation has potential therapeutic effects because it may enable them to gain control of physical processes that previously considered automatic responses of the autonomic nervous system (Tate and Milner, 2010). Therefore, biofeedback offers opportunities to improve accuracy during functional tasks, increase patients' engagement in their rehabilitation and reduce the need for ongoing contact with healthcare professionals to monitor the implementation of rehabilitation programs. The majority of biofeedback research has focused on the effects of biofeedback therapy in the treatment of upper and lower limb motor deficits in neurological disorders (Saita et al., 2018). A number of investigations have evaluated the effectiveness of force platform biofeedback during balance and mobility training tasks in older populations (Judge et al, 1993). Shivonen et al. (2004) demonstrated that visual feedback-based balance training using a force plate reduced the incidence of falls among frail older women living in residential care and enhanced performance of functional balancing tasks relevant to daily living.
In patients who have undergone thoracotomy, a significant asymmetry in the position of the center of gravity of the body is observed. This asymmetry causes the body to assume a right- or left-sided posture while maintaining balance in the upright position (Mraz et al., 2005). Furthermore, increases in both thoracic and lumbar curvatures are associated with a decrease in respiratory muscle strength, respiratory muscle thickness and some variables of lung function (Rahman, 2017). Therefore, we decided to introduce exercises on the stabilometric platform to a rehabilitation program for NSCLC patients after thoracic operations. The exercises on the stabilometric platform were safe and willingly performed by the patients. No complications occurred among the subjects of the study during the training period, and they were highly motivated to participate. After only a few sessions, the patients reported decreases in pain related to the thoracotomies. This effect was observed in the results of the SF-36 questionnaire, which revealed a significant reduction in the sense of pain. With the exception of GH, the EG exhibited improvements in all components of the PCS, and most of them (i.e., PF, BP, and PCS) were statistically significant. However, it should be stated that compared with the Control Group, only changes in Role Physical (RP) and Mental Health (MH) were statistically significant. We did not observe significant differences in the Fullerton test results obtained before and after rehabilitation between the EG and CG. All changes in the components of this test reflected improvements in fitness in both the EG and CG. In the EG, with the exception of the Back Scratch test results, significant improvements in all components of the Fullerton test were observed. Similar increases in fitness were observed in the control group.

According to the best of our knowledge, this is the first study using a stabilometric platform in pulmonary rehabilitation of NSCLC patients after thoracotomy. These preliminary results demonstrate that an enriched rehabilitation program involving stabilometric platform exercises made rehabilitation more attractive and did so without significant effects on the final results of rehabilitation in comparison with the controls. 
This study has several limitations. The

main ones are that the data were collected at a single rehabilitation center, and that the study sample was relatively small which is particularly important in the context of the estimation of the quality of life; such estimations require large populations to enrich not only the statistical, but also the clinical differences. Based on the overall account of our experiences in this study, we believe that 20-min daily sessions on the platform are too brief to elicit evident effects. Nonetheless, this type of exercise was well-tolerated by the

patients. We propound an increase in the number of 20-min sessions on the stabilometric platform to two per day. Exercises on the stabilometric platform should be preceded by stabilometric tests that will allow to program the exercises on the stabilometric platform according to individual needs. Finally, a two week period of exercises on stabilometric platforms seems to be too short to achieve significant improvements.

\section{Acknowledgements}

This study was supported by grant KNW-1-09/K/6/0 from the Medical University of Silesia, Poland.

\section{References}

Cattaneo D, Carpinella I, Aprile I, Prosperini L, Montesano A, Jonsdottir J. Comparison of upright balance in stroke, Parkinson and multiple sclerosis. Acta Neurol Scand, 2016; 133(5): 346-354

Dziadziuszko R. Malignant neoplasm of pleura and lung (C33-34). Polish National Cancer Registry, 2013. Available at: http://onkologia.org.pl/nowotwory-zlosliwe-oplucnej-pluca-c33-34/; accessed on 10.11.2017

Ferlay J, Soerjomataram I, Dikshit R, Eser S, Mathers C, Rebelo M, Parkin DM, Forman D, Bray F. Cancer incidence and mortality worldwide: sources, methods and major patterns in GLOBOCAN 2012. Int J Cancer, 2015; 136(5): 359-386

Holland A, Wadell K, Spruit M. How to adapt the pulmonary rehabilitation programme to patients with chronic respiratory disease other than COPD. EurRespir Rev, 2013; 22(130): 577-586

Jastrzebski D, Kozielski J, Banas A, Cebula T, Gumola A, Ziora D, Krzywiecki A. Quality of life during oneyear observation of patients with idiopathic pulmonary fibrosis awaiting lung transplantation. $J$ Physiol Pharmacol, 2005; 56(4): 99-106

Judge JO, Underwood M, Gennosa. Exercise to improve gait velocity in older persons. Arch Phys Med Rehabil, 1993; 74(4): 400-406

Mraz M, Sipko T, Godula A. Postural stability in thoracic surgery patients as assessed by posturographic tests. Fizjoterapia Polska, 2005; 5(1): 72-78

Oken MM, Creech RH, Tormey DC, Horton J, Davis TE, McFadden ET, Carbone PP. Toxicity And Response Criteria Of The Eastern Cooperative Oncology Group. Am J ClinOncol, 1982; 5(6): 649-655

Przybyłowski T, Tomalak W, Siergiejko Z, Jastrzębski D, Maskey-Warzęchowska M, Piorunek T, Wojda E, Boros P. Polish Respiratory Society guidelines for the methodology and interpretation of the 6 minute walk test (6MWT). PneumonolAlergol Pol., 2015; 83(4): 283-97

Rahman NN, Singh DK, Lee R. Correlation between thoracolumbar curvatures and respiratory function in older adults. Clinical Interventions in Aging, 2017; 12: 523-529

Rikli RE, Jones CJ. Functional Fitness Normative Scores for Community-Residing Older Adults, Ages 60-94. J Aging Phys Activ, 1999; 7(2): 162-181

Saita K, Morishita T, Arima H, Hyakutake K, Ogata T, Yagi K, Shiota E, Inoue T. Biofeedback effect of hybrid assistive limb in stroke rehabilitation: A proof of concept study using functional near infrared spectroscopy. PLoS One, 2018; 16; 13(1): e0191361 
Siekański K. Constructional solution of balance system for assessment and reeducation of proprioception and neuromuscular control of human motion system. Aktualne Problemy Biomechaniki, 2008; 2: 147-150

Sihvonen SE, Sipila S, Era PA. Changes in postural balance in frail elderly women during a 4-week visual feedback training: a randomized controlled trial. Gerontology, 2004; 50(2): 87-95

Stout N, Baima J, Swisher A, Winters-Stone K, Welsh J. A Systematic Review of Exercise Systematic Reviews in the Cancer Literature (2005-2017). PMER , 2017; 9(9S2): 347-384

Tate JJ, Milner CE. Real-time kinematic, temporospatial, and kinetic biofeedback during gait retraining in patients: a systematic review. Phys Ther, 2010; 90(8): 1123-1134

Vansteenkiste J, De Ruysscher D, Eberhardt WE, Lim E, Senan S, Felip E, Peters S. Early and locally advanced non-small-cell lung cancer (NSCLC): ESMO Clinical Practice Guidelines for diagnosis, treatment and follow-up. Ann Oncol, 2013; 24 (6): 89-98

Ware JE, Kosinski M, Gandek B. SF-36 Health Survey: Manual \& Interpretation Guide. Lincoln, RI: QualityMetric Incorporated; 2004

\section{Corresponding author:}

\section{Dariusz Jastrzebski MD, PhD}

Department of Lung Diseases and Tuberculosis, School of Medicine with the Division of Densitry, Medical University of Silesia, 41-803 Zabrze, Koziolka 1, Poland

Telephone: +48502038138

E-mail: darekjdr@poczta.onet.pl 